\title{
Reporting of Underrepresented Populations in Autism Treatment Studies Across 25 Years
}

\author{
Danai Kasambira Fannin \\ School of Allied Health \& Communicative Disorders, Northern Illinois University, DeKalb, IL, United States
}

Purpose: Eight language and autism related journals published between 1993 and 2017 were analyzed to identify trends in the inclusion of underrepresented populations in empirical studies with social skill, play, joint attention, and expressive language outcomes. Relationships among treatment outcomes, treatment settings, and underrepresented population types were explored.

Methods: All articles (12,381) were searched to identify intervention studies that included participants with autism and were classified by treatment target and setting $(N=291)$. The selected studies were then examined to determine if any participants were from underrepresented populations $(n=138)$ and categorized by type of underrepresented population.

Results: Most studies did not indicate demographic characteristics beyond disability, age, and gender; and few relationships between frequency of underrepresented population articles, treatment targets, or intervention setting emerged. Results indicated that demographic characteristics were often omitted, despite recommendations to report descriptive information.

Conclusions: Although inclusion of underrepresented populations has increased since 1993, treatment study results cannot yet be generalized to a more diverse population, due to the disproportionately low number of participants from racial/ethnic minority, multilingual, and low socioeconomic status groups reported and/or included in studies. The dearth of underrepresented participants suggests that conscious efforts to recruit and report them are still necessary.

Keywords: Autism, Research participants, Minorities, Language treatment

\section{INTRODUCTION}

The prevalence of Autism Spectrum Disorders (ASD) now exceeds other severe developmental disorders, making it a significant public health concern [1]. Timely administration of effective treatments is crucial [2] but knowledge of how to tailor treatments to particular children is incomplete. This is evidenced by inconsistent findings, where some children make expressive language gains, while others remain minimally verbal [3]. Further inquiry is necessary to the purpose of better customizing treatments to increase language gains across various populations.

Intervention approach is an active ingredient that may contribute to outcome variance, but other factors can alter response to the intervention. Proponents of dynamic systems theory (DST) posit that people have multiple, complex systems outside themselves, such as the home, neighborhood, community, school, workplace, and the people therein, that interact with the inherent developmental processes [4]. This theory ex-



Received: July 1, 2017

Revision: October 27, 2017

Accepted: December 20, 2017

Correspondence:

Danai Kasambira Fannin

360 Wirtz Hall, Northern Illinois University, DeKalb, IL, 60115, United States

Tel: +815-753-9126

Fax: +815-753-1664

E-mail:dfannin@niu.edu

This research was supported by Northern Illinois University Great Journeys Graduate Assistant Stipend Enhancement project, and the NIU College of Health and Human Sciences Support for Research Consultancy.

(C) 2017 The Korean Association of SpeechLanguage Pathologists

This is an Open Access article distributed under the terms of the Creative Commons Attribution Non-Commercial License (http:// creativecommons.org/licenses/by-nc/4.0/) which permits unrestricted non-commercial use, distribution, and reproduction in any medium, provided the original work is properly cited. 
plains, in part, the heterogeneity of symptoms characteristic of social communication disorders like ASD [5]. Thus, it follows that heterogeneity of how ASD presents itself might result in heterogeneity of the treatment response.

Dynamic systems theory supports the research focus of cultural neuroscience, which is the study of how culture can shape or be shaped by the mind, genes, and brain; and scientists are finding many similarities, but some differences, in how the brain functions across cultures [6]. Specifically, with respect to ASD, Dyches et al. [7] proposed that cultural factors can shape (a) when and how signs of ASD are defined and interpreted; (b) how families accept ASD diagnoses; and (c) whether there is a stigma associated with disability and to what degree it affects families. Accordingly, cultural family characteristics must be considered linked to how the intervention will be implemented and received. Thus, DST and cultural neuroscience establish why it would be prudent to analyze cultural characteristics as moderators to clarify for whom and under what conditions a treatment works [8].

Funding agencies have attempted to address the role of culture and DST in research. For example, the 1993 National Institutes of Health (NIH) Revitalization Act mandated reporting of demographics with prioritization of women and minorities [9]. As a result, more women were included, facilitating gains in knowledge of gender differences in disease presentation, symptoms, and treatment response [10]. Diversification by race/ethnicity has not been as successful, however, and disparities persist across a range of health indicators [11].

The fact that health disparities still exist and diversification by race/ethnicity has not been as effective as hoped 24 years later indicates limited (a) awareness of the feasibility of including minorities; (b) availability of demographic characteristics in researchers' datasets; (c) recognition of the importance of diverse samples; and (d) awareness of sample homogeneity in specific disciplines. All four factors are relevant to behavioral health studies targeting developmental disabilities, and the goal of the current study is to illustrate the aforementioned "limited awareness of sample homogeneity in specific disciplines," by describing the status of participant diversification among published ASD language intervention studies.

\section{Cultural Characteristics as Moderators}

Unfortunately, there are disparities in ASD diagnosis and intervention, primarily due to (a) homogeneous research samples $[12,13]$ and (b) overall health disparities blocking access to care. Furthermore, detailed descriptions of participants in
ASD treatment studies are lacking in general-not just for underrepresented populations (UP) [13,14]. The following factors may affect treatment and should therefore be properly represented and analyzed.

\section{Linguistic diversity}

Between 2006 and 2008, 55,076,078 individuals in the US over age 5 spoke languages other than English [15]. Thus, it seems reasonable that language treatment might need customization, depending on linguistic characteristics that may affect outcomes [16]. The scarcity of linguistically diverse participants limits generalization of efficacy trial results to linguistically varied populations. Thus, multilingual status should be identified and examined as a moderating cultural characteristic.

\section{Socioeconomic status}

It was once thought that ASD was specific to upper socioeconomic status (SES) populations [17]; but recent research suggests that this was due to ascertainment bias. In actuality, few researchers have taken such bias into account [18]. Yet the absence of an SES-ASD etiology link does not mean that SES cannot affect treatment. Indeed, general education literature shows that those from low SES homes can be exposed to different discourse styles and vocabulary than middle SES homes [19], signaling that SES should be analyzed in language studies. Moreover, growing income inequality $[45,46]$ makes the SES distinction increasingly important within racial/ethnic groups. One reason is that more formerly middle-SES European American (EA) are identifying themselves as low SES [47] and, as a result, can be part of this UP subtype of low SES. The assumption that EAs today are represented by the traditional participant characteristics is tenuous. Isolation from urban centers where research is often conducted makes rural, low-SES populations most underrepresented and some lowSES, rural regions are predominantly comprised of EAs [48].

\section{Race/ethnicity}

At the same time, we know that low SES and minority race/ ethnicity intersect, as evidenced by the poverty rate for African Americans (AA) being 27.6\%, Asians at $12.3 \%$ and, Latino Americans (LA) at 25.3\% [20]. Non-Hispanic Whites' poverty was the lowest, at $9.8 \%$. While there are no confirmed differences in ASD etiology by race/ethnicity or SES [21], a disparity in diagnosis along those demographic lines exists. Low SES and minority racial/ethnic status become cumulative risk factors, compounding the disparity, as demonstrated by the av- 
erage rate of ASD diagnoses being seven times lower for AAs and LAs than EA in a sample of Medicaid recipients [22,23]). Begeer, El Bouk, Boussaid, Terwogt, \& Koot [24] and Tek and Landa [25] attributed the disparity to subtle communication delays that lead to evaluations being missed or considered unremarkable by caregivers of particular cultures. Among non-EAs, it appears to take delays that are more significant to trigger evaluation, and, ultimately, intervention in non-EAs, indicating how background characteristics that may moderate outcomes might be cultural in nature [26]. When making spontaneous clinical judgments that would lead to further evaluation, healthcare providers have been found to generate ASD diagnoses in AA children less, which is concerning because some physicians use subjective questions or clinical judgment more than explicit standardized measures that reduce the racial/ethnic bias in ASD diagnosis [24]. The diagnosis disparity provides a partial explanation for underrepresentation of culturally and linguistically diverse (CLD) individuals in treatment studies, because later diagnoses result in later access to intervention studies.

\section{Relationship between CLD children, education, and assess- ment}

The juncture of SES, race/ethnicity, and linguistic diversity is significant because children from certain non-EA, low-SES homes are more likely to perform poorly on standardized language tests that are normed primarily on middle-SES, EA children who use Standard American English (SAE) [27]. The achievement gap between EAs and low-SES non-EAs occurs, in part, because of incongruences among socialization beliefs, parenting styles, and teaching style at school [28]. African American and LA families are in congruence with the mainstream teaching style [29] less often than EA families, and this information from the field of general education achievement and language assessment implies that families from various CLD groups might respond differently to (a) direct ASD assessment and treatment; (b) treatment conducted in the client's second language (English); (c) caregiver-mediated treatments; and (d) parent education models that were tested on monolingual, EA, middle-SES families. For this reason, researchers and interventionists must understand that the incongruence phenomenon may affect their practices. This understanding can only occur, however, with increased CLD participants in ASD studies and heightened reporting of demographics by those researchers who have been including CLD participants.

\section{Treatment Target and Setting}

Aside from cultural characteristics that might moderate treatment, the aforementioned home/school incongruence may be observed when a given cultural characteristic is either positively or negatively related to the treatment target. For instance, might a symbolic play treatment that is not as dependent on the interventionist's provision of language stimulation be effective for clients with limited English proficiency? Or are there certain social interventions that do not mirror the natural social interaction styles of another culture [30]? One step in the quest to answer these questions would be to identify any links between particular UP types and their inclusion in or exclusion from studies with particular treatment targets.

Treatment setting is another factor to consider when designing studies. Interventions conducted at a clinic may be prohibitive for people who cannot afford transportation, or those whose life obligations conflict with clinic treatment times [12]. As with treatment target inquiry, exploring setting as a possible reason for low UP participation must start with establishment of whether there are any relationships between certain UP types and settings. Treatment efficacy studies provide the evidence base for intervention [8], but a gap in knowledge of whether treatments are appropriate and effective for CLD people persists. This lacuna in the empirical base ultimately affects validation of ASD treatment efficacy overall. Hence, a fundamental step in mitigating this imparity would be to substantiate its existence by documenting the status of UP in ASD language treatment studies over time.

This study systematically documented reporting of racial/ ethnic minority, multilingual, and low-SES participants in empirical ASD treatment studies, examining those factors in relation to time periods, journal titles, and treatment settings and targets. One American Psychological Association (APA) journal (Journal of Consulting and Clinical Psychology $[J C C P])$, two American Speech-Language-Hearing Association (ASHA) journals (Journal of Speech, Language, and Hearing Research [JSLHR] and American Journal of SpeechLanguage Pathology [AJSLP]), and five ASD/developmental disability journals (Journal of Autism and Developmental Disorders [JADD], Focus on Autism and other Developmental Disabilities [FADD], American Journal on Intellectual and Developmental Disabilities [AJIDD], Autism Research and Treatment $[A R T]$, and Autism) were content analyzed. This design can highlight progress in the effort to include UP in studies by identifying trends in CLD participant inclusion. 
The research questions were:

1. What are the reporting practices of underrepresented populations (UP) for ASD treatment studies in eight journals over 25 years?

2. What are the associations between (a) UP type, (b) treatment setting, and (c) treatment targets?

\section{METHODS}

\section{Article Selection Criteria}

The United States Department of Education documented a 657\% increase in the autism rate between 1993 and 2003 [31], and the aforementioned NIH Revitalization Act went into effect in 1993; which corresponds with the first year analyzed for the current study. These journals were chosen because (a) people with ASD are a target population of readers, (b) these journals tend to publish studies with language and communication outcomes and, (c) most of the journals published all 25 years (yet, only two had ASD treatment studies the first six years). Other journals were excluded for lack of studies targeting language and communication. The AJIDD and Autism were established in 1997, so Autism had no data from 19931996, but, in the end, it contained the third highest amount of ASD treatment studies. Review articles, monographs, commentaries, essays, rejoinders, or other non-data-analytic reports were excluded.

Because the purpose of this study was to examine language treatment research that included participants with ASD, additional selection criteria were applied. A manual search of each issue allowed for identification of treatment targets, participant characteristics, and treatment settings. Articles were chosen for analysis if:

1. treatments targeted outcomes of expressive language (EL), social skills (Social), play skills (Play), or joint attention (JA) (alone or with other outcomes). Those with outcomes of only receptive language, reading, or written language were excluded.

2. participants had ASD, autism, autistic disorder, PDDNOS, or Asperger's syndrome. Articles discussing application to people with ASD but did not include any as participants were excluded.

3. the studies were (a) individual reports of research (i.e., not meta-analyses), (b) subsequent and distinct analyses of previous databases, (c) comparing effects of treatment to another condition, or (d) single-subject designs or case studies that involved measurement of treatment effects.
The titles and abstracts from 1993 to 2017 (12,381) were visually inspected by the authors and undergraduate research assistants (RA) for potential inclusion. When abstracts had vague study type descriptions, methods sections were read. All potential ASD treatment studies were then reviewed to determine if they met the aforementioned criteria, culminating in a database of 291 articles. Those articles were read in detail to code for treatment target, participant characteristics (low SES, race/ethnicity, multilingual), and setting.

\section{Coding}

Multilinguals were identified when researchers indicated the language background of participants. The researchers may have determined language status from participant self-report, or a language evaluation determining that the participant was multilingual. Therefore, if a coder read that participants were of Mexican descent but raised in the U.S., one could not assume they were multilingual. Hence, articles that omitted the language background of participants were not included in this category. Low SES was counted whenever researchers reported participants from low SES households. Socioeconomic status, race/ethnicity, and multilingual characteristics sometimes overlap; thus, they were counted multiple times. For example, a study that included racial/ethnic minorities was counted in the racial/ethnic category. Then, if those same participants were also low SES, that study was counted again in the low SES category. Racially/ethnically diverse participants were coded when researchers described participants as having non-EA race/ethnicity or when tables representing participant characteristics indicated non-EA participants.

The treatment target was defined by identifying treatment outcomes. Thus, a play-based treatment with an outcome of EL was coded as EL, not as Play. A play-based treatment with an outcome of improved symbolic play, however, would be coded as Play. As with UP type, studies could have been coded for multiple treatment targets. The location of treatment administration was coded when researchers reported where treatment was conducted. Multiple coding was allowed, for example, when part of the treatment was conducted at a clinic and part in homes, which resulted in a categorization of Clinic and Home. The following eight settings were coded:

1. Home was defined as a dwelling or group home that was not a residential school.

2. Clinic included outpatient facilities such as a private practice, hospital, or university lab. This represented in- 
tervention traditionally conducted in a room outside of schools.

3. General School was coded for treatment conducted in schools, including preschool, daycare, and residential schools.

4. Suburban School was coded when researchers reported that the school was in a suburb or rural area, including daycares, residential schools, and preschools.

5. Urban School (including preschools, daycares, and residential schools) was coded when researchers reported that the school was in an urban area.

6. Community included settings outside clinics, homes, or schools; such as neighborhood centers, restaurants, recreation facilities, or equestrian stables.

7. Setting Unspecified was coded when no setting type was reported.

8. University School was coded only when researchers explicitly reported the setting as a lab school, lab residential school affiliated with an institution of higher education, or school on a university campus.

\section{Training and Interrater Reliability}

Two post baccalaureate research assistants (RAs) who had used peer-reviewed publications for previous research were trained by the first author to select articles. The RAs and first author discussed criteria for selection of studies, and then practiced. During this process, they discussed disagreements, noted the reasoning for inclusion or exclusion for future reference, and then came to a consensus on whether to include the article. Once $90 \%$ agreement was established on practice articles, the RAs began independent selection. The RAs' selections were matched to those selected by the first author. Agreement was counted when all selected identical articles. Reliability conducted on four randomly selected years of each journal (18\%) and random reliability checks were performed periodically to ensure that the reliability remained $>90 \%$. Reliabilities were calculated as the number of agreed selections per journal/total articles selected, resulting in an interrater reliability of $90 \%$ (ranging from $50 \%$ to $100 \%$, with the $50 \%$ representing, in part, one coder who disagreed on one of three articles in $A R T$ ), indicating substantial agreement.

The first author trained a graduate RA also familiar with peer-reviewed articles and research methods to code the selected 291 articles by reviewing the coding system, practicing, and discussing discrepancies. The first author then conducted interrater reliability on $24 \%$ of the sample and once $>90 \%$ agreement was obtained in random checks, the graduate RA continued to code the rest of the sample. Few disagreements arose. For example, for studies conducted outside the U.S. (e.g., study conducted in Israel with Israeli and Russian participants), the first author decided that any non-American participants would be coded as an UP race/ethnicity, as any from foreign countries would be underrepresented due to their differing culture and likely multilingualism; had the study been conducted in the U.S. Coding reliabilities ranged from $78 \%$ to $100 \%$ with an agreement of $89 \%$.

\section{Analyses}

Using the SPSS statistical package version 24 (IBM, 2016) [32], relationships between UP articles and other variables like treatment settings, treatment targets, journal title, year, time period, and UP type were calculated. The data consisted of 291 articles from eight journals published between 1993 and 2017. 2017 articles were available through October so the majority of the year was analyzed. The UPArticles variable was dichotomous in a sense that it can take 1 or 0 based on whether the article reported UP. The covariates of interest were journal title (Journal; 1 to 8), year of publication (Year; 1993 to 2017), period of publication (Period; 1: 1993-1997, Period 2: 1998-2002, Period 3: 2003-2007, Period 4: 2008-2012, Period 5: 2013-2017), along with dichotomous variables indicating articles with treatment targeting improved play (TreatmentPlay; 1: yes, 0: no), joint attention (TreatmentJA; 1: yes, 0 : no), social skills (TreatmentSocial; 1: yes, 0 : no) and expressive language (TreatmentEL; 1: yes, 0: no). Indicator variables such as articles with participants of race/ethnicity other than EA (Race; 1: yes, 0: no), participants of low SES (LowSES; 1: yes, 0 : no), and participants who spoke more than one language (Multilingual; 1: yes, 0: no) were also included. Treatment settings were coded as Home; 1: yes, 0: no, Clinic; 1: yes, 0: no, SuburbSchool; 1: yes, 0: no, UrbanSchool; 1: yes, 0: no, GenericSchool; 1: yes, 0: no, Community; 1: yes, 0: no, UniversitySchool; 1: yes, 0: no, and SettingUnspecified; 1: yes, 0: no.

Due to uneven, smaller groups once subdivided by journal, treatment type, UP type, and year, nonparametric [33] Kruskal-Wallis analyses were used with a null hypothesis that there were no statistically significant differences between median frequency of UP articles by the factors of journal, year, Period, treatment type, setting, or UP type. The alpha value for significance was set at the $\leq .05$ level. The eta squared $\left(\eta^{2}\right)$ (nonparametric form of effect size) was calculated using chisquared divided by N-1 $\left(\chi^{2} / \mathrm{N}-1\right)$. Using Cohen's effect size in- 
tervals, Lenhard and Lenhard [34] interpret the magnitude of $\eta^{2}$ into intervals of: .004 to .059: small effect; .060 to .139: intermediate effect and; .140 and higher: strong effect. Effect sizes for follow up, Mann-Whitney $U$ tests [35] were calculated as the correlation coefficient $r(\mathrm{Z} / \sqrt{ } n)$ with 0.5 indicating a large effect, 0.3 as medium, and 0.1 as small.

\section{RESULTS}

Frequencies and percentages of variables were calculated and there were no qualifying ASD studies in 1994. Table 1 shows the frequencies and percentages of UP studies disaggregated by year and UP type.

A steady increase in diversity of participants was evident. However, the increase from Period 1 to Period 2 was based on starting with a single UP article in Period 1 . The increase in percentage of UP articles was $29 \%$ from Period 1 to Period 2, a decrease of $4 \%$ from Period 2 to 3 , a decrease of $3 \%$ from Period 3 to 4 , and a $38 \%$ increase from Period 4 to 5 . To illustrate how the reporting of UP moved in relation to relevant ASD studies, Figure 1 shows the total frequency of both ASD studies $(\mathrm{N}=291)$ and frequency of UP articles $(n=138)$ for each year. Although the UP line indicated fewer UP articles, a visual inspection of the graph showed the pattern of progression was similar.

Table 1. Number and Percentage of Studies Reporting UP by Year

\begin{tabular}{|c|c|c|c|c|c|c|c|c|c|}
\hline \multirow{2}{*}{ Year } & \multirow{2}{*}{ Total Studies } & \multirow{2}{*}{ Total UP } & \multirow{2}{*}{$\%$ UP } & \multicolumn{6}{|c|}{ Number and Percentage of UP Types } \\
\hline & & & & \# Race & $\%$ Race & \# Low SES & $\%$ Low SES & \# Multi & $\%$ Mult \\
\hline 1993 & 2 & 0 & $0 \%$ & 0 & $0 \%$ & 0 & $0 \%$ & 0 & $0 \%$ \\
\hline 1994 & 0 & 0 & $0 \%$ & 0 & $0 \%$ & 0 & $0 \%$ & 0 & $0 \%$ \\
\hline 1995 & 2 & 0 & $0 \%$ & 0 & $0 \%$ & 0 & $0 \%$ & 0 & $0 \%$ \\
\hline 1996 & 1 & 1 & $100 \%$ & 1 & $100 \%$ & 0 & $0 \%$ & 0 & $0 \%$ \\
\hline 1997 & 3 & 0 & $0 \%$ & 0 & $0 \%$ & 0 & $0 \%$ & 0 & $0 \%$ \\
\hline 1998 & 1 & 0 & $0 \%$ & 0 & $0 \%$ & 0 & $0 \%$ & 0 & $0 \%$ \\
\hline 1999 & 4 & 2 & $50 \%$ & 2 & $50 \%$ & 0 & $0 \%$ & 0 & $0 \%$ \\
\hline 2000 & 4 & 1 & $25 \%$ & 1 & $25 \%$ & 1 & $25 \%$ & 0 & $0 \%$ \\
\hline 2001 & 9 & 4 & $44 \%$ & 3 & $33 \%$ & 0 & $0 \%$ & 2 & $22 \%$ \\
\hline 2002 & 8 & 2 & $25 \%$ & 2 & $25 \%$ & 1 & $13 \%$ & 0 & $0 \%$ \\
\hline 2003 & 7 & 2 & $29 \%$ & 1 & $14 \%$ & 1 & $14 \%$ & 0 & $0 \%$ \\
\hline 2004 & 10 & 5 & $50 \%$ & 5 & $50 \%$ & 1 & $10 \%$ & 2 & $20 \%$ \\
\hline 2005 & 5 & 3 & $60 \%$ & 1 & $20 \%$ & 2 & $40 \%$ & 1 & $20 \%$ \\
\hline 2006 & 15 & 5 & $33 \%$ & 4 & $27 \%$ & 1 & $7 \%$ & 1 & $7 \%$ \\
\hline 2007 & 19 & 6 & $32 \%$ & 6 & $32 \%$ & 0 & $0 \%$ & 1 & $5 \%$ \\
\hline 2008 & 19 & 6 & $32 \%$ & 6 & $32 \%$ & 0 & $0 \%$ & 1 & $5 \%$ \\
\hline 2009 & 16 & 3 & $19 \%$ & 2 & $13 \%$ & 0 & $0 \%$ & 1 & $6 \%$ \\
\hline 2010 & 17 & 6 & $35 \%$ & 6 & $35 \%$ & 0 & $0 \%$ & 1 & $6 \%$ \\
\hline 2011 & 18 & 7 & $39 \%$ & 6 & $33 \%$ & 1 & $6 \%$ & 1 & $6 \%$ \\
\hline 2012 & 19 & 9 & $47 \%$ & 9 & $47 \%$ & 0 & $0 \%$ & 1 & $5 \%$ \\
\hline 2013 & 24 & 16 & $67 \%$ & 15 & $63 \%$ & 4 & $17 \%$ & 3 & $13 \%$ \\
\hline 2014 & 25 & 17 & $68 \%$ & 15 & $60 \%$ & 7 & $28 \%$ & 1 & $4 \%$ \\
\hline 2015 & 28 & 18 & $64 \%$ & 17 & $61 \%$ & 6 & $21 \%$ & 3 & $11 \%$ \\
\hline 2016 & 27 & 19 & $70 \%$ & 19 & $70 \%$ & 6 & $22 \%$ & 2 & $7 \%$ \\
\hline 2017 & 8 & 6 & $75 \%$ & 6 & $75 \%$ & 0 & $0 \%$ & 2 & $25 \%$ \\
\hline Total & 291 & 138 & $47 \%$ & 127 & $44 \%$ & 31 & $11 \%$ & 23 & $8 \%$ \\
\hline
\end{tabular}

$\# / \%$ Race= Number/Percentage of studies reporting race; \#/Low SES\% Race=Number/Percentage of studies reporting participants of low socioeconomic status; \#/\% Multi= Number/Percentage of studies reporting multilingual participants. 


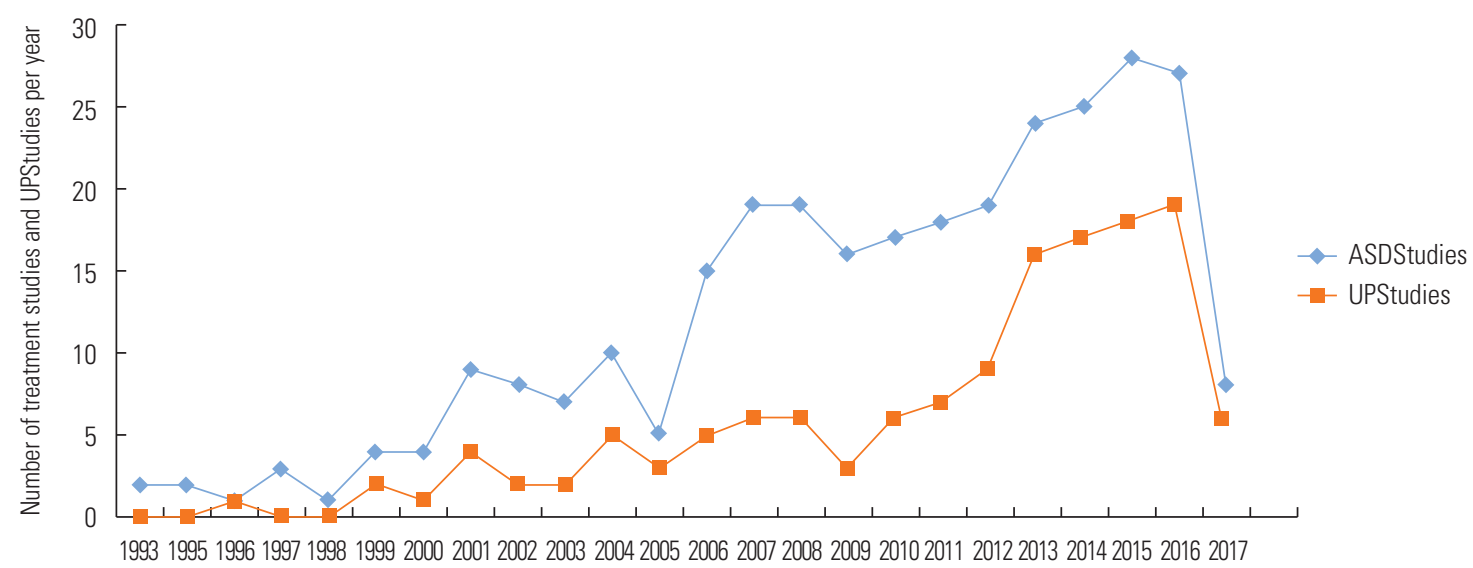

Figure 1. Number of treatment studies and UP studies per year.

ASDStudies=treatment studies targeting expressive language, joint attention, play, or social skills; UPStudies=treatment studies reporting race, low SES, or multilingual participants.

\section{UP Reporting by Journal}

One hundred thirty-eight of all 291 articles (47\%) reported UP. $J A D D$ had the highest number of treatment studies $(\mathrm{n}=152)$ with 85 reporting UP at 56\%. Autism had the second highest number of treatment studies $(\mathrm{n}=56)$ with 29 reporting UP at $52 \%$. FADD had 50 treatment studies with $28 \%(\mathrm{n}=14)$ reporting UP. Only $A R T$ reported zero UP, and the remaining journals reported two or three UP studies.

The Kruskal-Wallis test for Journal showed a significant difference in the frequency of UPArticles, $\chi^{2}(7)=19.395, p=0.007$, $\eta^{2}=0.07$ (intermediate effect). The Mann-Whitney $U$ test showed that $J A D D$ had significantly more UP articles than FADD $(U=2739.000, p=0.001, r=0.24)$ (small effect), AJIDD ( $U=487.000, p=0.028, r=0.17$ ) (small effect), and ART ( $U=$ 134.000, $p=0.027, r=0.18$ ) (small effect). FADD had significantly more UP articles than Autism ( $U=1067.000, p=0.013$, $r=0.24$ ) (small effect).

\section{UP Reporting by Year}

The Kruskal-Wallis test for Year showed a significant difference in the frequency of UPArticles, $\chi^{2}(23)=42.951, p=0.007$, $\eta^{2}=0.15$ (strong effect).

\section{Year 25}

The Mann-Whitney $U$ tests showed that Year 25 (2017) had significantly more UP articles than Year 5 (1997) $(U=3.000$, $p=0.034, r=0.64)$ (large effect), Year 15 (2007) $(U=43.000, p=$ $0.042, r=0.19)$ (small effect), Year 16 (2008) $(U=43.000$, $p=0.042, r=0.39$ ) (medium effect), and Year 17 (2009) ( $U=28.000, p=0.009, r=0.53$ ) (large effect).

\section{Year 24}

Year 24 (2016) had significantly more UP articles than Year 3 (1995) $(U=8.000, p=0.047, r=0.37)$ (medium effect), Year 5 (1997) $(U=12.000, p=0.018, r=0.43)$ (medium effect), Year 10 (2002) $(U=59.000, p=0.023, r=0.38)$ (medium effect), Year 11 (2003) $(U=55.000, p=0.046, r=0.34)$ (medium effect), Year 14 (2006) $(U=127.500, p=0.022, r=0.35)$ (medium effect), Year 15 (2007) ( $U=157.000, p=0.010, r=0.15)$ (small effect), Year 16 (2008) ( $U=157.000, p=0.010, r=0.38)$ (medium effect), Year 17 (2009) ( $U=104.500, p=0.001, r=0.49)$ (medium effect), Year 18 (2010) ( $U=149.000, p=0.024, r=0.34)$ (medium effect), and Year 19 (2011) $(U=166.500, p=0.038, r=0.31)$ (medium effect).

\section{Year 23}

Year 23 (2015) had significantly more UP articles than Year 5 (1997) ( $U=15.000, p=0.035, r=0.38$ ) (medium effect), Year 15 (2007) $(U=179.000, p=0.029, r=0.32)$ (medium effect), Year 16 (2008) ( $U=179.000, p=0.029, r=0.32)$ (medium effect), and Year 17 (2009) $(U=122.000, p=0.004, r=0.43)$ (medium effect).

\section{Year 22}

Year 22 (2014) had significantly more UP articles than Year 5 (1997) $(U=12.000, p=0.025, r=0.42)$ (medium effect), Year 10 (2002) $(U=57.000, p=0.035, r=0.37)$ (medium effect), Year 14 (2006) $(U=122.500, \mathrm{p}=0.035, r=0.33)$ (medium effect), Year 15 (2007) ( $U=151.000, p=0.018, r=0.36)$ (medium effect), Year 16 (2008) $(U=151.000, p=0.018, r=0.36)$ (medium effect), Year 17 (2009) ( $U=101.500, p=0.002, r=0.48)$ (medium 
effect), and Year 18 (2010) ( $U=143.000, p=0.039, r=0.32)$ (medium effect).

\section{Year 21}

Year 21 (2013) had significantly more UP articles than Year 5 (1997) $(U=12.000, p=0.030, r=0.42)$ (medium effect), Year 10 (2002) $(U=56.000, \mathrm{p}=0.043, r=0.36)$ (medium effect), Year 14 (2006) ( $U=120.000, p=0.045, r=0.32)$ (medium effect), Year 15 (2007) $(U=148.000, p=0.024, r=0.34)$ (medium effect), Year 16 (2008) ( $U=148.000, p=0.024, r=0.34)$ (medium effect), Year 17 (2009) $(U=100.000, p=0.003, r=0.46)$ (medium effect), and Year 18 (2010) $(U=140.000, p=0.050, r=0.30)$ (medium effect).

\section{UP Reporting by Period}

UP reporting was most frequent in Period 5, with $68 \%$ of all articles reporting UP, followed by Period 4 at $35 \%$ of the articles reporting UP. Figure 2 presents the percentage of total articles indicating UP by journal and 5-year period. JSLHR,
$A J S L P$, and JCCP did not report UP until Period 3 (2003+) and ART did not start until Period 4 (2008+). A consistent, rising trend of UP reporting was found for JADD. Meanwhile, Period 2 was the start of a plateau at approximately $20 \%$ of ASD studies for $F A D D$, and a decline in UP reporting for $A J I D D$.

The Kruskal-Wallis test for Period showed a difference in the frequency of UPArticles, $\chi^{2}(4)=32.140, p=0.001, \eta^{2}=0.11$ (intermediate effect). The Mann-Whitney $U$ test showed that Period 5 had more UP articles than Period $1(U=200.000, p=$ $0.002, r=0.29$ ) (small effect), Period 2 ( $U=972.000, p=0.002$, $r=0.27$ ) (small effect), Period $3(U=2184.000, p=0.001$, $r=0.29$ ) (small effect), and Period $4(U=3338.000, p=0.001$, $r=0.33$ ) (medium effect).

\section{Associations among Treatment Type, UP Type, and Treatment Setting}

Table 2 shows how most AJIDD (80\%), Autism (75\%), JADD (69\%), and FADD (68\%) articles targeted social skills (Social), while all articles in both ASHA journals targeted expressive

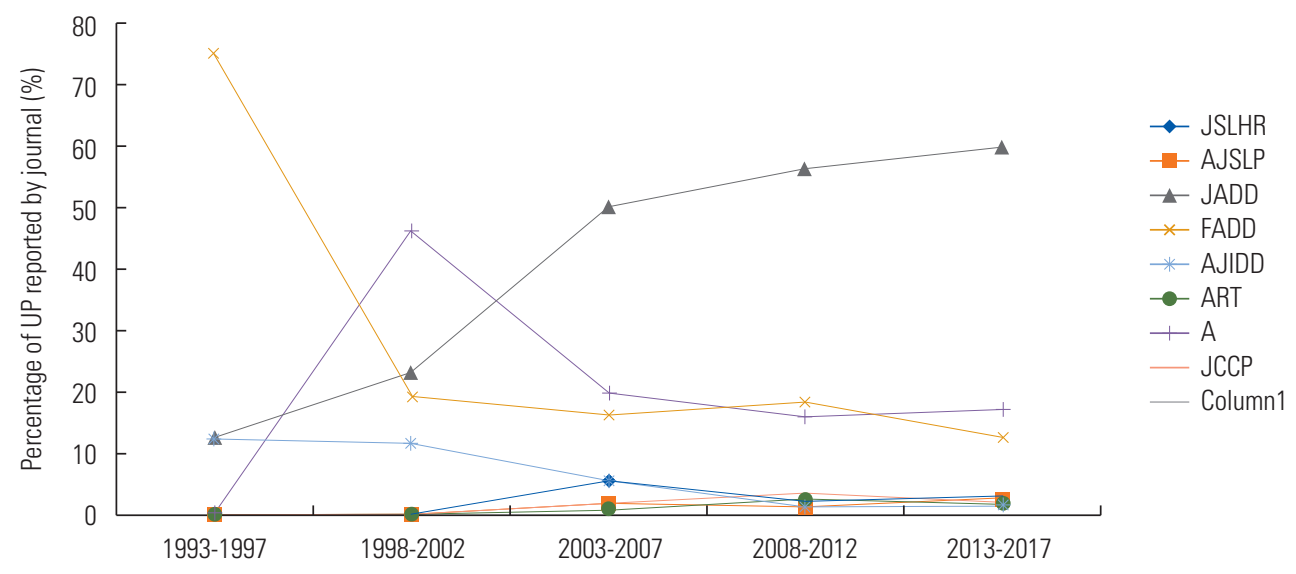

Figure 2. Percentage of Underrepresented Participant (UP) reporting.

JSLHR= Journal of Speech, Language, and Hearing Research; AJSLP=American Journal of Speech Language Pathology; JADD= Journal of Autism and Developmental Disorders; FADD = Focus on Autism and Other Developmental Disabilities; AJIDD=American Journal of Intellectual and Developmental Disabilities; ART =Autism Research and Treatment; $A=$ Autism; JCCP = Journal of Counseling and Clinical Psychology.

Table 2. Number and Percentage of Designated Topics in Qualifying Studies Across Eight Journals

\begin{tabular}{|c|c|c|c|c|c|c|c|c|c|c|c|c|c|c|c|c|}
\hline \multirow{2}{*}{ Topic } & \multicolumn{2}{|c|}{$J S L H R$} & \multicolumn{2}{|c|}{$A J S L P$} & \multicolumn{2}{|c|}{$J A D D$} & \multicolumn{2}{|c|}{$F A D D$} & \multicolumn{2}{|c|}{$A J I D D$} & \multicolumn{2}{|c|}{$A R T$} & \multicolumn{2}{|c|}{ Autism } & \multicolumn{2}{|c|}{ JCCP } \\
\hline & $(n=8)$ & $\%$ & $(n=5)$ & $\%$ & $(n=152)$ & $\%$ & $(n=50)$ & $\%$ & $(n=10)$ & $\%$ & $(n=4)$ & $\%$ & $(n=56)$ & $\%$ & $(n=6)$ & $\%$ \\
\hline Play & 1 & 13 & 1 & 20 & 16 & 11 & 6 & 12 & 0 & 0 & 0 & 0 & 17 & 30 & 2 & 33 \\
\hline JA & 0 & 0 & 0 & 0 & 14 & 9 & 1 & 2 & 1 & 10 & 0 & 0 & 10 & 18 & 4 & 67 \\
\hline Social & 2 & 25 & 1 & 20 & 105 & 69 & 34 & 68 & 8 & 80 & 3 & 75 & 39 & 71 & 2 & 33 \\
\hline $\mathrm{EL}$ & 8 & 100 & 5 & 100 & 57 & 38 & 23 & 46 & 8 & 80 & 1 & 25 & 21 & 38 & 4 & 67 \\
\hline
\end{tabular}

$J S L H R=$ Journal of Speech, Language, and Hearing Research; AJSLP =American Journal of Speech Language Pathology; JADD= Journal of Autism and Developmental Disabilities; FADD=Focus on Autism and other Developmental Disabilities; AJIDD=American Journal on Intellectual and Developmental Disabilities; ART =Autism Research and Treatment; JCCP = Journal of Consulting and Clinical Psychology; JA = Joint Attention; EL=Expressive Language. 
language (EL). The topic that appeared alone most often $(\mathrm{N}=291)$ was Social $(\mathrm{n}=194)$. For the studies that had more than one topic, however, Social and EL $(\mathrm{n}=50)$ comprised the most common combination, with 16 of those being UP studies (32\%). The next highest combination was Social and Play $(\mathrm{n}=24)$, and seven had UP (29\%). Eight ASD studies had Social and joint attention (JA) outcomes, and four of those (50\%) had UP. Eighteen ASD studies combined EL and Play, and six of those included UP (33\%). Nine ASD studies targeted EL and JA and five had UP (56\%). Lastly, 10 studies had Play and JA and seven of those had UP (70\%). The Kruskal-Wallis test for treatment types showed no significant differences in the number of articles that reported Race, LowSES, or Multilingual.

\section{UP type}

Two by two contingency table analyses were first used to ascertain associations among UP articles, UP types, treatment settings, and treatment types. For UPArticles and UP types, the tests were asymptotic because the tests require a great number of observations. Articles not related to UPArticles (UPArticles $=0$ ) only corresponded to articles with EA participants $($ Race $=0)$ and this relation holds by the way the variables are defined. For conditional probability, every article with non-EA participants was related to UPArticles, whereas, only 7\% (11 over 164) of those with EA participants also included UP. The proportion of UP articles with no Low SES participants was $41 \%$ (107 over 260), and the proportion of UP articles with no multilingual participants was $43 \%$ (115 over 268). Fisher's exact tests for Race, Low SES, and Multilingual produced two sided p-values at .00, confirming strong evidence of association between UP type and UPArticles. Therefore, no further analyses between UP type and UP articles were conducted.

\section{Treatment setting}

Kruskal-Wallis tests showed no differences in the frequency of UPArticles for Clinic, $\chi^{2}(1)=0.093, p=0.76$; Home, $\chi^{2}(1)=$ 0.258, $p=0.61$; SuburbSchool, $\chi^{2}(1)=0.009, p=0.93$; UrbanSchool, $\chi^{2}(1)=2.156, p=0.14$; UniversitySchool, $\chi^{2}(1)=0.596$, $p=0.44$; SettingUnspecified, $\chi^{2}(1)=0.033, p=0.86$; GenericSchool, $\chi^{2}(1)=0.375, p=0.54$, or Community, $\chi^{2}(1)=0.527, p=$ 0.47 . When considering UP type and treatment setting, a Kruskal-Wallis test for all eight settings showed no differences in the number of articles that reported Race or Multilingual. LowSES, however, showed a difference where it was reported significantly more in UrbanSchool settings $\chi^{2}(1)=17.612$, $p=0.000, \eta^{2}=0.06$ (intermediate effect).

\section{Treatment type}

A 2 by 2 contingency table for TreatmentPlay and UPArticle showed that $40 \%$ ( 17 over 43 ) of Play articles had UP; whereas, $49 \%$ (121 over 248) of non-Play articles related to UP. Conditional probabilities ( $49 \%$ and $40 \%$ ) were not substantially different and 2-sided $p$-values from the contingency table were more than 0.05. The Kruskal-Wallis test for TreatmentPlay showed no significant difference in the frequency of UPArticles, $\chi^{2}(1)=1.255, p=0.26$.

UPArticle observations were cross classified based on whether they contained Social treatment; resulting in $44 \%$ ( 85 over 194) of Social studies having UP, while the remaining 52 UP articles (54\%) did not target Social. These proportions were not sufficiently significant to show a strong association between TreatmentSocial and UPArticle, as all the two-sided p-values were higher than 0.1. The Kruskal-Wallis test for TreatmentSocial showed no significant difference in the frequency of UPArticles, $\chi^{2}(1)=2.752, p=0.10$.

A 2 by 2 contingency table for TreatmentEL and UPArticle showed the proportion of UP in EL articles was $45 \%$ ( 57 over 127), while the proportion of UP articles with non-EL targets was $49 \%$ (80 over 163). The Kruskal-Wallis test for Treatmen$t E L$ showed no significant difference in the frequency of UPArticles, $\chi^{2}(1)=0.503, p=0.48$.

UPArticle was cross-classified based on whether JA was targeted. Few had JA targets and, of those studies, $60 \%$ (18 over 30) had UP; whereas $46 \%$ (120 over 261) of the non-JA articles had UP. The Kruskal-Wallis test for TreatmentJA showed no significant difference in the frequency of UPArticles, $\chi^{2}(1)=$ $2.115, p=0.15$.

\section{DISCUSSION AND CONCLUSIONS}

Scientists have been designing language interventions for ASD, but questions remain about their efficacy and what external factors influence the effectiveness of intervention. Several published language treatments exist, and clinicians who employ evidence-based practice are using these to plan the most appropriate interventions for various populations [13]. However, the evidence base for determining whether these treatments work for everyone is not as strong as the evidence for monolingual, English-speaking, middle-SES EAs. As such, the gaps in research considering CLD populations should be examined to encourage more inclusion of CLD participants, 
and identify specific recruitment targets. This study supports the contentions of Oh et al. [10] that, despite movement toward more inclusive samples and an increase in ASD treatment studies, the number of UPs reported is still low. Results were also consistent with recent research on UP in ASD [12, 13], and this systematic examination revealed some significant findings.

\section{ASD Treatment Study Trends Over Time}

The frequency of ASD treatment studies targeting language did increase over the 25 years, but 291 out of 12,381 (2\%) articles still represents a small proportion. The rate of ASD diagnosis had been considered stable until the late 1990s [36] but prevalence has increased remarkably since then: from 1 in 150 in 2000 to 1 in 68 at present, representing an annual increase of 6-15\% [1]. Increased diagnoses have driven funding agencies to request proposals for clinical research, which may account for the surge in treatment studies in Period 5; reflecting the urgency to find evidence-based ASD interventions.

\section{UP Trends Over Time}

Though ASD treatment studies for language increased, only $47 \%$ reported UP--consistent with previous findings [8,12,13, $37,38]$. This could be due to variable recognition of the importance of reporting and analyzing the diversity of samples, or minimal awareness of sample homogeneity by scientists who target language, social skills, play, and joint attention. Reporting of UP in these language based studies became inconsistent in the latter part of Period 2 through Period 4 and there are few similar publications on UP in ASD treatment studies against which to compare current results. A similar pattern of change, however, was indicated by McLoyd and Randolph's [39] examination of Child Development up to 1980, and Graham's [40] investigation of 20 years of psychology studies in American Psychological Association journals that included AAs. Specifically, inclusion of racial/ethnic minorities in psychology journals (wherein some ASD empirical studies can be found) has fluctuated but remained low. This is similar to the current findings for race/ethnicity, as well as low SES and multilingual reporting, inasmuch as multilingualism and low SES are more likely to intersect with racial/ethnic minority status [22].

\section{UP Trends by Treatment Target}

The intervention targets conform to the notion that core deficits of ASD include joint attention, socialization, and play, which are all associated with expressive language development [41]. Thus, researchers are grounding their treatment targets in the theory that bolstering pivotal skills such as symbolic play and JA $[42,43]$ should augment EL development. However, in light of the confounding factors of (a) potential clinician/client mismatch in cultural socialization expectations, (b) clinician/client home language mismatch, and (c) clinician/client mismatch in expected play skills, styles, and routines, more inquiry is needed to ensure generalization of language interventions to CLD populations.

Just as there were no associations between treatment targets and UP articles overall, there were no significant relationships between EL, JA, Social, or Play outcomes when UP types were separated. There are no previous data to corroborate these results, indicating the need for further investigation into any relationships between specific treatment targets and different UP types. One reason UP type should be studied in relation to treatment target is that demographic factors often cooccur in various combinations [20]. Thus, disentangling UP types would be essential to identifying demographic moderators of treatment targets.

The increasing diversity of the U.S. and known differences in discourse styles across cultures $[19,28]$ calls for an examination of whether ASD language treatment may or may not need customization for particular populations [16]. Because it is largely unknown whether specific UP types moderate ASD treatment $[8,44]$ or particular UP characteristics are correlated with other factors, such as treatment setting or target, an analysis beyond simple frequency of UP inclusion is essential.

\section{UP Trends by Journal Title}

Certain treatment types including UP were specific to journal title. Among the eight journals, JADD, FADD, and Autism published the most. ART had fewer studies because it started publishing in 2010 and included a broad range of non-treatment research, or treatment studies (e.g., drug studies) that did not meet the current study's criteria. Only JADD had continuous growth in UP studies over the 25 years. Autism's reporting of UP increased to Period 2, but diverged from the other journals' increasing or plateau patterns through Period 5 , as indicated by a significant decline in UP reporting going into Period 3. The current analyses do not allow an explanation for this trend, and Pierce et al. [13] could not identify a particular event in the area of ASD research that would account for the inconsistency in their analyses of race/ethnicity reporting in Autism, FADD, and $J A D D$. Detailed examination 
of any subject matter themes or special topics, however, might reveal that ASD treatment studies with other outcomes were published in $F A D D$ that were excluded from these analyses. For example, some ASD treatment studies used outcomes that did not qualify them for inclusion-e.g., verbal comprehension, listening comprehension, making transitions between activities, and behavior-and one study evaluated outcomes for typical peers, not those with ASD. Thus, a decrease in reported UP may be a function of the current study's selection criteria.

\section{UP Trends by Treatment Settings}

There were no trends in UP studies by treatment setting type, which is consistent with Crosland et al. [14], who found no trend in their study of research across 15 years. More than $60 \%$ of their studies were conducted in an inpatient hospital setting or clinic, with less than $40 \%$ being administered in communities, homes, or schools; but, over time, there was a slight increase in studies being conducted in the latter settings. In the current study, $38 \%$ of those that reported settings (not including Unspecified Setting $[\mathrm{n}=12]$ ) were conducted in clinics, $20 \%$ in homes, $17 \%$ in suburban/urban/university schools, $20 \%$ in generic schools, and $5 \%$ in the community. Crosland et al. [14] did not analyze types of interventions, and they included more types of outcomes, as evidenced by their inclusion of studies in Journal of Applied Behavior Analysis with independent variables of educational, behavioral, or psychological interventions, which the current study excluded. Hence, an exact, apples-to-apples comparison of Crosland's study to the current study was not possible.

\section{Limitations}

Although the database was small because of the nature of the variables, the large sample of approximately 12,381 studies was a methodological strength, as it gives greater confidence to assert that, while the number of ASD intervention studies has increased over the past two decades, there is still a relative paucity of UP among those studies. Other strengths include the wider timespan, the higher number of journals, and the coding of UP types beyond race/ethnicity.

The primary limitation was that some of the journals did not yield many ASD treatment studies overall. The authors ensured inclusion of $J A D D, F A D D$, and Autism, however, which are known for publishing these types of studies. Analyses of the other journals illustrated the state of ASD treatment studies in publications with respect to language interventions.
However, the low number of qualifying studies in those journals limited statistical analyses - and some of the variables (e.g., treatment target, treatment setting, and UP types) did not have enough observations to conduct reliable parametric analyses at a $p \leq .05$ significance level. Future studies can address the noted limitations by increasing the sample size via a shift in years studied and, perhaps, replacement of some of the journals with newer ones focused on ASD.

\section{Implications}

Cultural differences in how people play, communicate, and use language show the importance of reporting all types of demographic information, especially now that there are more samples large enough to potentially detect an effect based on a single UP type. Despite widespread knowledge of the confounding factors of language background, SES, and racial/ ethnic background $[8,16,26,44]$, however, many language focused studies still do not consider SES in their research design or they exclude participants whose primary language is not English. The deficiencies in UP representation in treatment studies have important implications for clinicians who serve a population increasing in both ASD diagnoses and diversity, and in the pursuit of evidence-based practice for all, recommendations have been made to recruit more inclusive samples. As a result, more researchers may indeed be including UP, but they may still be omitting detailed participant descriptions, prohibiting definitive proof that the treatment effects apply to UP. According to Pierce et al. [13]:

Even when no differences are found across ethnic variables, generalizations of intervention outcomes are more plausible within or across samples, despite the possibility that some variance regarding race and ethnicity may still exist (Dyches et al. 2004; Trembath et al. 2005; Wallis and Pinto-Martin 2008). Moreover, relevant data may also emerge when a comprehensive analysis of ethnicity and race factors are conducted. Liptak et al. (2008) noted that while there were NSD (no significant differences) in how ethnic groups accessed medical services, children diagnosed with ASD were more likely to have private health insurance and were identified by a higher SES than other children. Including such information may urge researchers to examine other influences associated with diversity that may emerge. (p. 9).

This problem of under-reporting might be mitigated through better enforcement of reporting across journals through reviewer requests for demographic details during the 
peer review process.

While carefully considering and adapting approaches to recruitment and treatment setting, treatment time, and treatment models may improve and facilitate UP participation, measures can also be taken to encourage researchers to include UP and to report demographic characteristics in ways that can address questions related to treatment for UP.

In order to make recruitment of UP a more deliberate decision, journals can require researchers to report detailed participant demographics [40]. It is likely that more studies in the sample included UP than were reported; however, it is impossible to obtain an accurate representation of participant demographics if researchers do not report that information. Beyond simply reporting demographics, the manner in which the information is collected and how it is reported are vital considerations. For example, a direct and common way to designate SES is inquiring about household income on a demographic questionnaire and then assigning poverty status based on national definitions of poverty. This may not always be effective, however, even when participants are assured that questionnaires are anonymous, because provision of that information is often optional. Yet there are other ways to determine SES like social class measures using occupational prestige, marital status, educational level, or retired/employed status $[49,50]$. Indirect measures, such as school records of receiving reduced-cost lunch or participation in an education program such as Head Start, can also indicate whether the participants are from low-SES communities without participants declaring their annual income.

Ideally, demographic factors would be reported for specific participants, as these facilitate description of the sample with increased accuracy [44]. For instance, a few authors from the current sample reported that schools had a higher percentage of students who receive reduced-cost lunch, but this did not tell the reader whether the study participants were among those students. Others reported that the treatment was conducted in an urban school and a suburban school, but there are affluent schools in urban areas that have a school environment and resources similar to suburban schools. In short, to understand whether the treatment worked for low-SES participants, indicators for specific participants are preferable.

In summary, the results provide evidence of the persistent lack of UP in ASD treatment studies with communication/ language targets across two decades and eight peer reviewed journals; and/or a lack of reporting of UP. Future research should examine other types of ASD treatment studies that have included UP $[13,14]$ to ascertain possible reasons for success or failure in including UP, as well as whether initiatives to enforce thorough demographic reporting at the peer review level would result in improved reporting. Without more participation of CLD participants, the evidence base for ASD intervention cannot assuredly be generalized beyond middle SES, monolingual EAs. Better representation is therefore critical to the promotion of good science, effective and efficient treatments, economics, and social justice [10]. Researchers must take this a step further, however, and improve reporting practices that can ultimately facilitate better implementation science.

\section{ACKNOWLEDGMENTS}

This study was funded by the Northern Illinois University Great Journeys Graduate Assistant Stipend Enhancement project, and the College of Health and Human Sciences Support for Research Consultancy. The author would like to thank Connie Kasari for her guidance; Colin Ong-Dean and Peter Rosenbaum for helping proofread the manuscript and the Research Assistants; Nancy Creel, Jasmine Donley, and Asra Yousufuddin for their hard work and dedication to the project.

\section{REFERENCES}

1. Center for Disease Control (CDC). Autism and Developmental Disabilities Monitoring Network. Retrieved from: http://www.cdc. gov/ncbddd/autism/addm.html. 2014.

2. Dingfelder HE, Mandell DS. Bridging the research-to-practice gap in autism intervention: An application of diffusion of innovation theory. Journal of Autism and Developmental Disorders.2011;41 (5): 597-609.

3. Kasari C, Lawton K. New directions in behavioral treatment of autism spectrum disorders. Current Opinion in Neurology. 2010;23: 137-143.

4. De Bot K, Lowie W, Verspoor M. A dynamic systems approach to second language acquisition. Bilingualism: Language and Cognition. 2007;10(1):7-21.

5. Hwa-Froelich DA. Theoretical foundations and introduction. In Hwa-Froelich DA, editor. Social Communication Development and Disorders. New York: Psychology Press, 2014. p. 3-19.

6. Westby C. Social neuroscience. In Hwa-Froelich DA, editor. Social Communication Development and Disorders. New York: Psychology Press, 2014b. p. 20-49.

7. Dyches TT, Wilder K, Sudweeks RR, Obiakor RE, Algozzine B. Multicultural issues in autism. Journal of Autism and Developmental Disorders. 2004;34:211-222.

8. Miranda J, Bernal G, Lau A, Kohn L, Hwang W, LaFromboise T. 
State of the science on psychosocial interventions for ethnic minorities. Annual Review of Clinical Psychology. 2006;1(1):113-142.

9. United States Congress. National Institutes of Health Revitalization Act of 1993: Act to Amend the Public Health Service Act to Revise and Extend the Programs of the National Institutes of Health, and for Other Purposes. Public Law 103-43. Washington, DC. 1993;103-143.

10. Oh SS, Galanter J, Thakur N, Pino-Yanes M, Barcelo NE, White MJ, et al. Diversity in clinical and biomedical research: A promise yet to be fulfilled. PLoS Medicine, 2015;12(12):1-9. doi: 10.1371/journal.pmed.1001918.

11. United States Department of Health and Human Services, Office of Minority Health. National Partnership for Action to End Health Disparities. The National Plan for Action Draft as of February 17, 2010 [Internet]. Chapter 1: Introduction. Retrieved from http://www.minorityhealth.hhs.gov/npa/templates/browse.aspx?\&lvl=2\&lvlid=34

12. Hilton CL, Fitzgerald RT, Jackson KM, Maxim RA, Bosworth CC, Shattuck PT, et al. Brief report: Under-representation of African Americans in autism genetic research: A rationale for inclusion of subjects representing diverse family structures. Journal of Autism and Developmental Disorders. 2010;40(5):633-709.

13. Pierce NP, O'Reilly MF, Sorrells AM, Fragale CL, White PJ, Aguilar $\mathrm{JM}$, et al. Ethnicity reporting practices for empirical research in three autism-related journals. Journal of Autism and Developmental Disorders. 2014;44(7):1507-1519.

14. Crosland KA, Clarke S, Dunlap G. A trend analysis of participant and setting characteristics in autism intervention research. Focus on Autism and Other Developmental Disabilities. 2012;28(3):159165.

15. United States Census Bureau. Detailed languages spoken at home and ability to speak English for the population 5 years and over for the United States: 2006-2008. Retrieved from http://www.census. gov/hhes/socdemo/language/

16. Magnusson C, Rai D, Goodman A, Lundberg M, Idring S, Svensson A, et al. Migration and autism spectrum disorder: Population based study. The British Journal of Psychiatry. 2012;201(2):109115.

17. Schopler E, Andrews CE, Strupp K. Do autistic children come from upper-middle-class parents? Journal of Autism and Developmental Disorders. 1979;9(2):139-152.

18. Bhasin KT, Schendel D. Sociodemographic risk factors for autism in a US metropolitan area. Journal of Autism and Developmental Disorders. 2007;37:667-677.

19. Qi CH, Kaiser AP, Milan S, Hancock T. Language performance of low income, African American and European American preschool children on the Peabody Picture Vocabulary Test-III. Language, Speech, and Hearing Services in Schools. 2006;37:1-12.

20. United States Census Bureau. Income, poverty, and health insurance coverage in the United States, 2011: Current population reports. Population profile of the United States: America at the close of the 20th century. Retrieved from http://www.census.gov/prod /www/abs/p60.html.

21. Fombonne E. Epidemiological surveys of autism and other pervasive developmental disorders: An update. Journal of Autism and Developmental Disorders. 2003;33:365-382.
22. Gutman LM, Sameroff AJ, Cole, R. Academic growth curve trajectories from 1st grade to 12th grade: Effects of multiple social risk factors and preschool child factors. Developmental Psychology. 2003;39(4):777-790.

23. Mandell DS, Morales KH, Xie M, Lawer LJ, Stahmer AC, Marcus SC. Age of diagnosis among Medicaid-enrolled children with autism. Psychiatric Services. 2010;61:822-829.

24. Begeer S, El Bouk S, Boussaid W, Terwogt, MM, Koot HM. Underdiagnosis and referral bias of autism in ethnic minorities. Journal of Autism and Developmental Disorders. 2009;39(1):142-148.

25. Tek S, Landa RJ. Differences in autism symptoms between minority and non-minority toddlers. Journal of Autism and Developmental Disorders. 2012;42(9):1967-1973.

26. Machalicek W, O'Reilly MF, Beretvas N, Sigafoos J, Lancioni G, Sorrells A, et al. A review of school based instructional interventions for students with autism spectrum disorders. Research in Autism Spectrum Disorders. 2008;2:395-416.

27. Craig HK, Washington JA. An assessment battery for identifying language impairments in AA children. Journal of Speech, Language, and Hearing Research. 2000;43:450-463.

28. Barbarin O, Jean-Baptiste E. The relation of dialogic, control, and racial socialization practices to early academic and social competence: Effects of gender, ethnicity, and family socioeconomic status. American Journal or Orthopsychiatry. 2013;83(2):207-217.

29. Barbarin O, Downer J, Odom E, Head D. Home-school differences in beliefs, support, control and their relations to the competence of children in Publicly Sponsored Pre-K Programs. Early Childhood Research Quarterly. 2010;25:368-372.

30. Sutton A. Language acquisition theory and AAC intervention. Perspectives on Augmentative and Alternative Communication. 2008;17:56-61.

31. Lilienfeld SO, Arkowitz H. Is there really an autism epidemic? Retrieved from http://www.scientificamerican.com/article/is-therereally-an-autism-epidemic/.

32. IBM Corp. IBM SPSS Statistics for Windows, Version 24.0. Armonk, NY: IBM. 2016.

33. Dallal GE. Nonparametric statistics. In The little handbook of statistical practice. Retrieved from http://www.jerrydallal.com/ LHSP/npar.htm. 2010.

34. Lenhard W, Lenhard A. Calculation of Effect Sizes. Dettelbach (Germany): Psychometrica. Retrieved from: https://www.psychometrica.de/effect_size.html. 2016.

35. Fritz CO, Morris PE, Richler J. Effect size estimates: Current use, calculations, and interpretations. Journal of Experimental Psychology. 2011;141:2-18.

36. Yeargin-Allsopp M, Rice C, Karapurkar T, Doernberg N, Boyle C, Murphy C. Prevalence of autism in a US metropolitan area. JAMA. 2003;289(1):49-55.

37. Artiles AJ, Trent SC, Kuan L. Learning disabilities empirical research on ethnic minority students: An analysis of 22 years of studies published in selected refereed journals. Learning Disabilities Research \& Practice. 1997;12(2):82-91.

38. Kasari C, Patterson S. Interventions addressing social impairment in autism. Current Psychiatry Reports. 2012;6:713-725.

39. McLoyd V, Randolph S. Secular trends in the study of Afro-Ameri- 
can children: A review of child development, 1936-1980. Monographs of the Society for Research in Child Development. 1985;50 (4-5, Serial No. 211).

40. Graham S. "Most of the Subjects were White and Middle-Class": Trends in published research on African Americans in selected APA journals, 1970-1989. American Psychologist. 1992;47(5):629639.

41. Toth K, Munson J, Meltzoff AN, Dawson G. Early predictors of communication development in young children with autism spectrum disorder: Joint attention, imitation and toy play. Journal of Autism and Developmental Disorders. 2006;36:993-1005.

42. Dykstra JR, Boyd BA, Watson LR, Crais ER, Baranek GT. The impact of the Advancing Social-Communication and Play (ASAP) intervention on preschoolers with autism spectrum disorder. Autism: The International Journal of Research and Practice. 2012;16, 27-44.

43. Paparella T, Goods KS, Freeman S, Kasari C. The emergence of nonverbal joint attention and requesting skills in young children with autism. Journal of Communication Disorders, 569-583. http://dx.doi.org/10.1016/j.jcomdis.2011.08.002.

44. Shanawani HH, Dame, LL, Cook-Deegan RR. Non-reporting and inconsistent reporting of race and ethnicity in articles that claim associations among genotype, outcome, and race or ethnicity.
Journal of Medical Ethics: Journal of the Institute of Medical Ethics. 2006;32:72-728.

45. Corak M. Income inequality, equality of opportunity, and intergenerational mobility. The Journal of Economic Perspectives. 2013;27(3):79-102.

46. Fry R, Taylor P. A rise in wealth for the wealthy; Declines for the lower 93\%. Retrieved from http://www.pewsocialtrends.org/2013/ 04/23/a-rise-in-wealth-for-the-wealthydeclines-for-the-lower-93/.

47. Morin R, Motel S. A third of Americans now say they are in the lower classes. Retrieved from http://www.pewsocialtrends. org/2012/09/10/a-third-of-americans-now-say-they-are-in-thelower-classes/.

48. Burchinal MR, Vernon-Feagans L, Cox M, Key Family Life Project Investigators. Cumulative social risk, parenting, and infant development in rural low-income communities. Parenting, Science, and Practice.2008;8(1):41-69.

49. Warner WL, Meeker M, Eells K. Revised occupational rating scale from Warner, Meeker, and Eells' Index of status characteristics. (1949) In Miller DC, editor. Handbook of research design and social measurement. (5th ed.). Newbury Park, CA: Sage Publications. 1991.

50. Hollingshead AA. Four-factor index of social status. Unpublished manuscript, Yale University, New Haven, CT. 1975. 